\title{
Cannabinoids in the Treatment of Neurological Disorders
}

\author{
Orrin Devinsky $^{1} \cdot$ Benjamin J. Whalley ${ }^{2} \cdot$ Vincenzo Di Marzo $^{3}$
}

Published online: 3 September 2015

(C) The American Society for Experimental NeuroTherapeutics, Inc. 2015

The force of the recent explosion of largely unproven and unregulated cannabis-based preparations on medical therapeutics may have its greatest impact in the field of neurology. Paradoxically, for 10 millennia this plant has been an integral part of human cultivation, where it was used for its fibers long before its pharmacological properties. With regard to the latter, cannabis was well known to healers from China and India thousands of years ago; Greek and Roman doctors during classic times; Arab doctors during the Middle Ages; Victorian and Continental physicians in the nineteenth century; American doctors during the early twentieth century; and English doctors until 1971 when a variety of nonevidence-based remedies were removed from the British Pharmaceutical Codex. However, cannabis-based or cannabis-derived medicines have been almost entirely absent from American medicine and barely present in European and Asian pharmacopeias during the twenty-first century [1].

Dronabinol (Marinol) is the only cannabinoid-based medicine that is approved by the US Food and Drug Administration, with an indication for loss of appetite associated with weight loss in patients with AIDS and those receiving chemotherapy [2]. Dronabinol is the synthetic active enantiomer of the plant-derived cannabinoid, $\Delta^{9}$-tetrahydocannabinol

Orrin Devinsky

od4@nyu.edu

1 Department of Neurology, Comprehensive Epilepsy Center, New York University School of Medicine, New York, NY 10016, USA

2 School of Pharmacy, The University of Reading, Whiteknights, Reading, Berkshire RG6 6AP, UK

3 Institute of Biomolecular Chemistry, Consiglio Nazionale delle Ricerche, Via Campi Flegrei 34, Comprensorio Olivetti, 80078 Pozzuoli, NA, Italy
(THC) and works via cannabinoid type $1\left(\mathrm{CB}_{1}\right)$ and type 2 $\left(\mathrm{CB}_{2}\right)$ receptors $\left(\mathrm{CB}_{1} \mathrm{R}\right.$ and $\mathrm{CB}_{2} \mathrm{R}$, respectively), where it acts as a partial agonist [3]. In more than 20 European and other countries outside the US, nabiximols (Sativex; GW Pharmaceuticals, Cambridge, UK) is approved to treat spasticity in patients with multiple sclerosis [4]. Nabiximols is a mixture extracts of two varieties of the cannabis plant, one predominantly containing THC and the other cannabidiol (CBD). Interestingly, and contrary to popular misconception, $\mathrm{CBD}$ is not a potent ligand at either $\mathrm{CB}_{1} \mathrm{R}$ or $\mathrm{CB}_{2} \mathrm{R}$, although occasionally it can functionally modulate the consequences of mammalian endocannabinoid system stimulation in which the 2 cannabinoid receptors play a pivotal role [5]. In fact, CBD has several noncannabinoid receptor molecular targets and thus exemplifies the diverse pharmacology of the 500 discrete components of the cannabis plant, of which nearly 100 have cannabinoid-like chemical structures.

The relative scarcity of proven cannabis-based therapies is not due to data that show that cannabinoids are ineffective or unsafe, but rather reflects a poverty of medical interest and a failure by pharmaceutical companies arising from regulatory restrictions compounded by limits for patent rights on plant cannabinoid-containing preparations that have been used medicinally for millennia, as is the case for most natural products. In some Western countries, funding to study, establish, and prevent adverse effects of recreational cannabis use, such as addiction and cognitive and behavioral disorders, has far outpaced basic or clinical scientific research in this area. This is not surprising, as in the USA, funding by the National Institute of Drug Abuse on research into cannabis abuse has dramatically exceeded that into cannabis therapeutics by all other National Institutes of Health institutes combined [6], despite the societal change favoring the deregulation of recreational cannabis use in the USA. 
Phytocannabinoids-cannabinoids synthesized by plants - have a diverse range of biological effects from calcium homeostasis to antioxidant and anti-inflammatory properties and many more [7]. Scientific interest in the pharmacology of cannabis constituents has steadily grown over the last 50 years since the isolation and synthesis of THC and CBD (isolated in 1940, characterized in 1963, and synthesized in the late 1960s) [8-11]. The action of THC at $\mathrm{CB}_{1} \mathrm{R}$ and the endocannabinoid system [including $\mathrm{CB}_{1} \mathrm{R}$ and $\mathrm{CB}_{2} \mathrm{R}$, their endogenous ligands (the endocannabinoids), and metabolic enzymes for endocannabinoid biosynthesis and inactivation] were discovered in the late 1980s and early 1990s, and has been the most intensively studied aspect of cannabis pharmacology $[12,13]$. A PubMed search (May 2015) identified 19, 095 references on 'cannabinoid', 13,919 on 'cannabis', 6740 on 'endocannabinoid', 6579 on THC, and 1351 on CBD. These results suggest a wealth of information in the scientific and medical literature, but the vast majority is from basic science using in vitro or animal studies, which justifies caution if effects are being extrapolated to humans. The clinical data on cannabis therapeutics are meager and the vast majority are formed by surveys or small studies that are underpowered and/or suffer from multiple methodological flaws, often by virtue of limited research funding for nonaddiction-focused studies. Thus, we know relatively little about the clinical efficacy of cannabinoids for the various neurological disorders for which historical nonscientific and medical literature have advocated its use.

The challenge now for scientific medicine is that the "horse is out of the barn". A wave of cannabis legislation, driven in part by medical claims and also by a changing societal view of recreational cannabis use, has washed across the USA, Uruguay, and Portugal, and will likely spread elsewhere as pressure from patients, their parents, and advocates demand access for its use in a range of disorders that can impair or destroy quality of life, or kill. Randomized placebo-controlled studies have been the main road to governmental approval of drugs and devices for the last half century and remain the gold standard by which efficacy is objectively judged. Yet cannabis has effectively bypassed that road by virtue of a 'perfect storm' made up of medical lack of interest, commercial unpalatability, societal acceptance of the naturalistic fallacy, and urgent unmet clinical need. A diverse range of cannabis plant strains, rich in different proportions of the 100 or so phytocannabinoids that have been identified, have been bred, and complex and often hazardous processing techniques devised to increase and extract specific components such as CBD or THC-acid, with highly variable results. These products are being used by consumers - from children with severe epilepsy to patients with chronic pain or insomnia or anxiety or cancer. There is a growing group of lay experts who profess knowledge of the correct mixture of constituents (e.g., "You need at least $2 \%$ THC with the CBD to stop seizures", or
"CBD will not work if the child is taking leviteracetam") who lack data and, often via association with alternative medicine schools of thought, do not feel beholden to the medical establishment's basic demand for objective proof of safety and efficacy. We are moving back to days of Paracelsus and alchemy but multiplied by the echo chamber of social media and growing scientific illiteracy [14].

We believe that when nutritional supplements are recommended for either a specific disease (e.g., Parkinson disease) or general health benefits (e.g., cognitive function), there should be valid data to support those claims - data that are independent of bias created by placebo responses, financial gains, or other factors. Government should help to protect people from harm - whether the harm comes from spending money on products that do not deliver on their promise or have the potential to cause adverse effects that do not outweigh their harmful effects. Our governments make it illegal to sell a television that does not work but have allowed the dietary supplement industry to make innumerable health claims, which have no evidential basis and, in many cases, have been even refuted by scientific data. We therefore applaud the efforts of governmental agencies to identify fraudulent claims about compounds purported to contain CBD in which CBD is wholly absent or present in miniscule amounts.

We hope that this issue of Neurotherapeutics will serve to mark the bounds of verifiable scientific knowledge of cannabinoids in the treatment of neuropsychiatric and neurological disorders. At the same time, our contributors have also helped identify areas for future research, as well as the strategies needed to move our base of knowledge forward. We hope that this volume will help to accelerate the pace of the appropriately focused and productive research and double-blind placebo-controlled randomized trials to the point at which the care of patients is informed by valid data and not just anecdote.

We are pleased to have gathered many of the world's experts together on the basic biology of cannabinoids, as well as their potential role in treating neurologic and psychiatric disorders. Dr. Di Marzo briefly introduces the endocannabinoid system. Dr. Whalley explains the molecular pharmacology of cannabidiol in neurological disorders, while Dr. Justinova discusses the biology of endocannabinoids. Dr. Gloss reviews the currently available cannabinoid products and the challenges facing our interpretation of the data associated with their clinical use. Drs Sachs, McGlade, and Yurgelun-Todd provide a general overview of the safety and toxicity of cannabinoids. Dr. Notcott reviews the clinical use of cannabinoids for the treatment of multiple sclerosis. Drs Stephens, Arjmand, and Shabani review the use of cannabinoids in the treatment of tremors. Drs Fernandez-Ruiz, Martinez-Orgado, and Moro review the use of cannabinoids in the treatment of neurodegenerative disorders and stroke. Dr. Hohmann reviews the use of cannabinoids in pain treatment. Drs Jutras-Aswad and Hurd 
review the use of cannabinoids in drug abuse treatment. Drs Blessing, Steenkamp, Marmar, and McCabe review cannabinoid use to treat anxiety. Dr. Goff reviews the risks and benefits of utilizing CBD as a treatment for schizophrenia. Drs Maccarrone and Chakrabarti explore the therapeutic potential of cannabinoids in treating autism. Dr. Koppel reviews the use of cannabinoids in treatment for dyskinesia, dystonia, and tics. Drs Rosenberg, Tsien, Whalley, and Devinsky review the use of cannabinoids in epilepsy.

Required Author Forms Disclosure forms provided by the authors are available with the online version of this article.

\section{References}

1. Whalley BJ. Cannabis in the management and treatment of seizures and epilepsy: A scientific review. AHP. Available at: http://www. herbal-ahp.org/documents/press_releases/AHP\%20Therapeutic\% 20Compendium-Cannabis\%20Epilepsy $\% 20$ and $\% 20$ Seizures $\%$ 20Scientific\%20Review.pdf. Accessed June, 2015.

2. Kramer JL. Medical marijuana for cancer. Cancer J Clin 2015;65: 109-122.

3. Pertwee RG. The diverse $\mathrm{CB} 1$ and $\mathrm{CB} 2$ receptor pharmacology of three plant cannabinoids: delta9-tetrahydrocannabinol, cannabidiol and delta9-tetrahydrocannabivarin. Br J Pharmacol 2008;153:199215.
4. Sastre-Garriga J, Vila C, Clissold S, Montalban X. THC and CBD oromucosal spray (Sativex $\left.{ }^{\circledR}\right)$ in the management of spasticity associated with multiple sclerosis. Expert Rev Neurother 2011;11: 627-637.

5. McPartland JM, Duncan M, Di Marzo V, Pertwee RG. Are cannabidiol and $\Delta(9)$-tetrahydrocannabivarin negative modulators of the endocannabinoid system? A systematic review. Br J Pharmacol 2015;172:737-753.

6. Harris D. Researchers find study of medical marijuana discourages. New York Times January 10, 2010. Web. Available at: http://www. nytimes.com/2010/01/19/health/policy/19marijuana.html?_r=2. Accessed June, 2015.

7. Pertwee R. Handbook of cannabis. Oxford University Press, Oxford, 2014.

8. Gaoni Y, Mechoulam R. Isolation, structure, and partial synthesis of an active constituent of hashish. J Am Chem Soc 1964;86:1646-1647.

9. Adams R, Pease DC and Clark JH. Isolation of Cannabinol, Cannabidiol and Quebrachitol from Red Oil of Minnesota Wild Hemp. J Am Chem Soc 1940;62:2194-2196.

10. Lerner M. Marihuana: Tetrahydrocannabinol and Related Compounds. Science 1963;140:175-176.

11. Michoulam R, Shvo Y and Hashish I. The structure of cannabidiol. Tetrahedron. 1963;19:2073-2078.

12. Maccarrone M, Bab I, Bíró T, Cabral GA, Dey SK, Di Marzo V, et al. Endocannabinoid signaling at the periphery: 50 years after THC. Trends Pharmacol Sci. 2015;36:277-296.

13. Pertwee RG. Ligands that target cannabinoid receptors in the brain: from THC to anandamide and beyond. Addict Biol. 2008b;13:147159.

14. Duncan DE. 216 Million Americans Are Scientifically Illiterate (Part I). MIT Technology Review. 21 Feb 2007. N.p. Web. 22 Mar 2014. 